\title{
Extremely Nonperturbative Nonlinearities in GaAs Driven by Atomically Strong Terahertz Fields in Gold Metamaterials
}

\author{
C. Lange,${ }^{1,}{ }^{*}$ T. Maag, ${ }^{1}$ M. Hohenleutner,${ }^{1}$ S. Baierl, ${ }^{1}$ O. Schubert, ${ }^{1}$ E. R. J. Edwards, ${ }^{2}$ \\ D. Bougeard, ${ }^{1}$ G. Woltersdorf, ${ }^{2}$ and R. Huber ${ }^{1}$ \\ ${ }^{1}$ Department of Physics, University of Regensburg, 93040 Regensburg, Germany \\ ${ }^{2}$ Department of Physics, Martin-Luther-University Halle-Wittenberg, 06120 Halle, Germany \\ (Received 18 April 2014; revised manuscript received 14 July 2014; published 26 November 2014)
}

\begin{abstract}
Terahertz near fields of gold metamaterials resonant at a frequency of $0.88 \mathrm{THz}$ allow us to enter an extreme limit of nonperturbative ultrafast terahertz electronics: Fields reaching a ponderomotive energy in the $\mathrm{keV}$ range are exploited to drive nondestructive, quasistatic interband tunneling and impact ionization in undoped bulk GaAs, injecting electron-hole plasmas with densities in excess of $10^{19} \mathrm{~cm}^{-3}$. This process causes bright luminescence at energies up to $0.5 \mathrm{eV}$ above the band gap and induces a complete switch-off of the metamaterial resonance accompanied by self-amplitude-modulation of transmitted few-cycle terahertz transients. Our results pave the way towards highly nonlinear terahertz optics and optoelectronic nanocircuitry with subpicosecond switching times.
\end{abstract}

DOI: 10.1103/PhysRevLett.113.227401

PACS numbers: 78.20.-e, 42.65.Ky, 42.65.Sf, 72.20.Ht

Intense, phase-locked light pulses in the terahertz spectral range have opened up an exciting arena for field-sensitive nonlinear optics [1-22]. For a given peak electric field $\mathcal{E}$, the low carrier frequency $\omega_{\mathrm{THz}}$ gives rise to a potentially large ponderomotive energy $U_{p}=$ $e^{2} \mathcal{E}^{2} / 4 m \omega_{\mathrm{THz}}^{2}$, which quantifies the cycle-averaged quiver energy of a free electron of mass $m$. This situation promises a new quality of nonperturbative light-matter interaction at the boundary of terahertz optics and highspeed electronics.

For frequencies between 0.5 and $3 \mathrm{THz}$, optical rectification [7-10] has enabled transients with peak field amplitudes in excess of $1 \mathrm{MV} / \mathrm{cm}$ [10]. Using such an electromagnetic pulse as an alternating bias, terahertzdriven carrier multiplication in doped semiconductors [12] and graphene [14] has been demonstrated. Strong terahertz fields have also been used to drive spectacular nonlinear intraband dynamics of quasiparticles, such as field ionization of impurity states [13] or excitons, electronhole recollisions, and high-order sideband generation [11]. In these experiments, the terahertz bias facilitates tunneling of bound electrons through potential energy barriers, which correspond to binding energies between a few $\mathrm{meV}$ and several $10 \mathrm{meV}$ [Fig. 1(a)] [11,13,23].

A new limit of nonperturbative nonlinearities is expected if terahertz amplitudes approach atomically strong fields. As depicted in Fig. 1(b), for typical semiconductors like GaAs, hypothetical terahertz amplitudes of $12 \mathrm{MV} / \mathrm{cm}$ would cause a transient potential energy drop by the fundamental energy gap $E_{g}=1.4 \mathrm{eV}$ over distances as small as two unit cells, and field-induced interband tunneling should start to dominate. Using a band model [24], we estimate that under such conditions more than $10^{20}$ valence electrons per $\mathrm{cm}^{3}$ may undergo Zener tunneling through the fundamental band gap into the conduction band within a time window of only $100 \mathrm{fs}$, possibly inducing even degenerate carrier populations in intrinsic bulk semiconductors [25].

Phase-locked light pulses featuring amplitudes in the $100 \mathrm{MV} / \mathrm{cm}$ range have become available at carrier frequencies of multiple $10 \mathrm{THz}$ [3]. This breakthrough has enabled ultrafast biasing of bulk solids in an unprecedented high-field limit, where a coherent interplay between nonresonant interband polarization and intraband Bloch oscillations generates terahertz high-harmonic radiation [6]. The diffraction limit of focusing, however, has precluded comparably high fields at frequencies as low as $1 \mathrm{THz}$ where yet larger ponderomotive potentials $U_{p}$ combined with photon energies orders of magnitude below electronic interband resonances could pave the way to quasistatic biasing. Custom-tailored metamaterials are a promising concept for overcoming the diffraction limit. Indeed, field enhancement in metamaterials has been exploited to induce a metal-insulator phase transition in $\mathrm{VO}_{2}$ by a terahertz transient with a carrier frequency below $1 \mathrm{THz}$ [34], and to evoke strong terahertz nonlinearities through terahertz-driven intervalley scattering and impact ionization $[22,35]$.

Here, we combine metamaterials with $\mathrm{MV} / \mathrm{cm}$-scale fewcycle sub-1-THz transients to explore an extreme regime of nonperturbative nonlinearities in the well-defined laboratory of the bulk semiconductor GaAs. Atomically strong fields exceeding $10 \mathrm{MV} / \mathrm{cm}$ and ponderomotive potentials in the $\mathrm{keV}$ range lead to strong carrier generation across the band gap which exceeds the central terahertz photon energy by a factor of 390. Ultrabroadband near-infrared and visible interband luminescence emerges, and a strong terahertz nonlinearity results on the single-cycle time scale. 


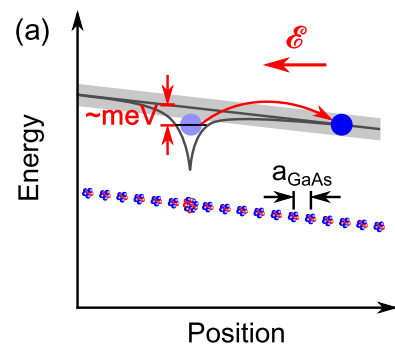

(c)

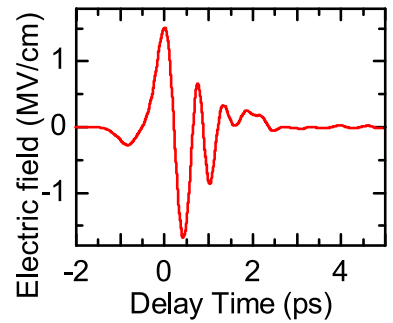

(b)

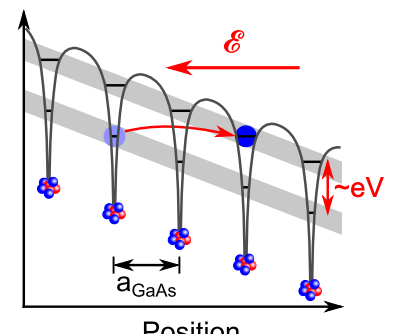

(d)

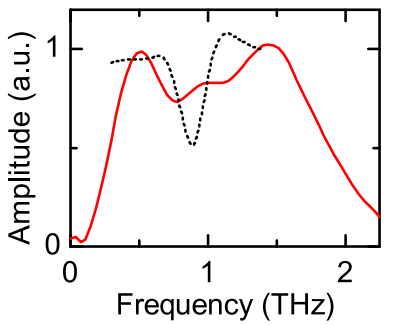

(e)

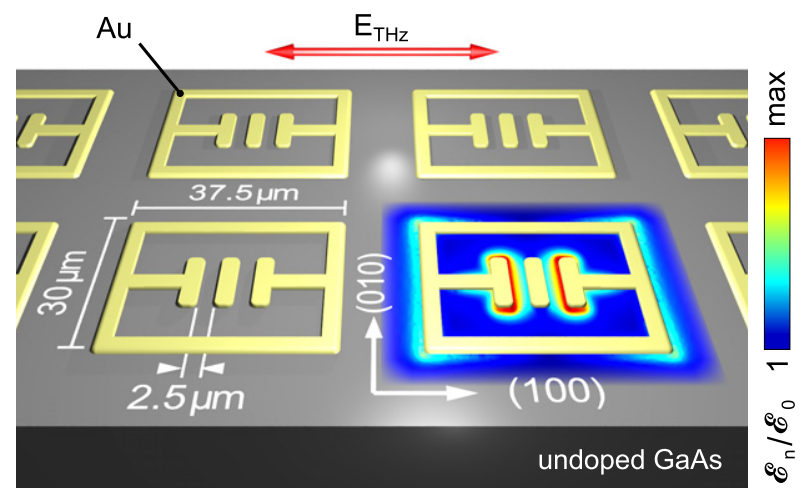

FIG. 1 (color online). (a) A terahertz field of $\mathcal{E}=500 \mathrm{kV} / \mathrm{cm}$ sufficiently skews the conduction band edge (solid line) of GaAs (lattice constant $a_{\mathrm{GaAs}}$ ) to drive field ionization of shallow impurity states (blue sphere). (b) Under an atomically strong bias of $\mathcal{E}=12 \mathrm{MV} / \mathrm{cm}$ the energy landscape is so strongly tilted that valence- and conduction-band-like states of nextneighboring unit cells become energetically degenerate and massive interband Zener tunneling should set in. (c) Far-field waveform of phase-locked few-cycle $\mathrm{THz}$ transients (amplitude $1.5 \mathrm{MV} / \mathrm{cm}$ ) with (d) spectrum (red line) and normalized metamaterial transmission (dotted curve). (e) Geometry of the resonator forming the unit cell of the terahertz metamaterial on (100)-oriented GaAs as indicated. The red double arrow marks the terahertz polarization for optimal coupling to the fundamental resonator mode. The near-field enhancement of the structure at the resonance frequency is highlighted as a color plot for the lower right resonator. This map has been calculated by FDFD simulation for a plane $300 \mathrm{~nm}$ below the surface of the substrate of sample A.

Near-infrared light pulses centered at a wavelength of $800 \mathrm{~nm}$ (pulse energy $3.5 \mathrm{~mJ}$, pulse duration $35 \mathrm{fs}$ ) are derived from a Ti:sapphire laser amplifier. We employ tilted-pulse front optical rectification [9] in a cryogenically cooled $\mathrm{LiNbO}_{3}$ crystal to generate phase-locked terahertz transients [Fig. 1(c)] with a peak field of $\mathcal{E}_{0}=1.5 \mathrm{MV} / \mathrm{cm}$ in the far-field focus on the sample, and an amplitude spectrum ranging from 0.1 to $2.5 \mathrm{THz}$ [Fig. 1(d)]. For electro-optic detection of the terahertz waveform, the transmitted beam is imaged onto a $10-\mu$ m-thick ZnTe detection crystal and gated by 35-fs near-infrared pulses.

The unit cell of our metamaterials is given by a planar gold resonator [Fig. 1(e)] featuring a $2.5-\mu \mathrm{m}$-wide double capacitive gap, symmetrically closed by inductive loops on each side. This miniature $R L C$ circuit of outer dimensions of $37.5 \times 30 \mu \mathrm{m}^{2}$ is designed for a resonance frequency of $0.88 \mathrm{THz}$. Millimeter-sized, two-dimensional arrays of identical resonators made of 150-nm-thick gold are fabricated by electron-beam lithography, thermal evaporation, and lift-off processes. The gold structure and a 3-nm-thick chromium adhesion layer are created either directly on a substrate of undoped (100)-oriented GaAs (sample A) or on top of an additional $\mathrm{Al}_{2} \mathrm{O}_{3}$ layer of a thickness of $t=100 \mathrm{~nm}$ (sample B) or $t=300 \mathrm{~nm}$ (sample C). Figure 1(e) shows the typical distribution of the electric near field in the unit cell of the metamaterial, on resonance, as computed by finite-difference frequency-domain (FDFD) calculations. In the capacitive gap region of the structure, the electric field is strongly enhanced, whereas the magnetic field enhancement is negligible (not shown) and thus not considered further.

Near the capacitive stripes of our metamaterials, timedomain simulations predict an enhancement factor between the near $\left(\mathcal{E}_{n}\right)$ and the far field $\left(\mathcal{E}_{0}\right)$ of up to 35 for our terahertz pulse shape [25]. For the highest terahertz far fields attained in our experiment this enhancement would correspond to $\mathcal{E}_{n}>50 \mathrm{MV} / \mathrm{cm}$ if no nonlinearities occurred. To date, there is no calibrated procedure to characterize such enormously large fields. Established near-field measurements relying on electro-optic sampling [36,37], tunneling currents [38], or Franz-Keldysh oscillations [39] are not characterized for atomically strong fields in which even the concept of a rigid electronic band structure is pushed to the limits of its validity. Possible characterization methods may, however, arise from the following observations.

We illuminate our structure with intense terahertz pulses and map the metamaterial plane onto a silicon CCD camera or a grating spectrometer using a microscope objective. The strong near-field enhancement in the metamaterial manifests itself in a spectacular way: Resonators located in the terahertz focus $\left(\mathcal{E}_{0}=1.5 \mathrm{MV} / \mathrm{cm}\right)$ exhibit bright visible electroluminescence (EL) in GaAs. False-color microscope images of the spectrally integrated luminescence intensity emanating from a selected area of metamaterial B and close-up views of individual terahertz resonators in all three samples are shown in Figs. 2(a)-2(d), respectively. For a direct comparison, a map of the calculated local field enhancement is shown in Fig. 2(e). The spatial distribution of the emitted radiation correlates strongly with the local field: Most of the luminescence originates from the region close to the stripes of the capacitive double-gap area, where 

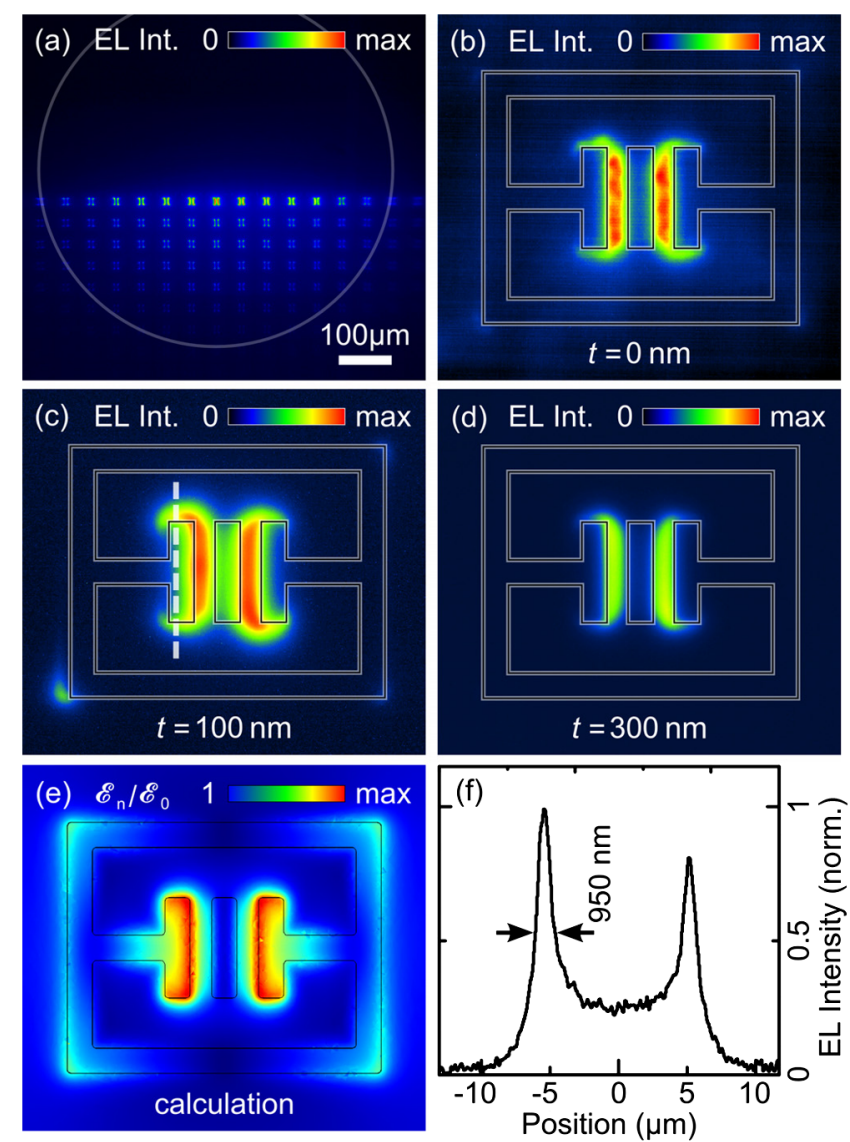

FIG. 2 (color online). (a) Terahertz-induced EL from the metamaterial array. The terahertz focus (FWHM outlined by white circle) is positioned at the horizontal border between the metamaterial field (lower half) and the unstructured GaAs substrate (upper half), where no emission is observed. (b) Microscopic view of the EL intensity from a single resonator (sample A, thickness of the insulating layer $t=0 \mathrm{~nm}$ ) driven by the terahertz transient of Fig. 1(c) $\left(\mathcal{E}_{0}=1.5 \mathrm{MV} / \mathrm{cm}\right)$. The outline of the resonator is superimposed on the luminescence image as a guide to the eye. (c),(d) EL intensity images from resonators of samples B and C, with $t=100 \mathrm{~nm}$ and $t=300 \mathrm{~nm}$, respectively. (e) Color map of calculated near-field distribution indicating the modulus of the electric field amplitude at the resonance frequency of $0.88 \mathrm{THz}$, in a plane $300 \mathrm{~nm}$ below the semiconductor surface. (f) The EL intensity profile across the capacitor plate [dotted line in (c)] demonstrates a spatial resolution better than $950 \mathrm{~nm}$.

the field enhancement is largest, while emission is virtually absent in unstructured areas of the substrate. Typical spatial profiles of the EL intensity [cf. Fig 2(f)] exhibit features of a FWHM of less than $950 \mathrm{~nm}$ and allow for indirect tracing of the local terahertz field amplitude.

The microscopic origin of the strong light emission is apparent from its spectral shape. Figure 3(a) shows typical EL intensity spectra obtained for a series of terahertz amplitudes. For $\mathcal{E}_{0}=120$ and $380 \mathrm{kV} / \mathrm{cm}$, the luminescence extends from the direct band gap of GaAs at $E_{g}=1.42$ up to $1.7 \mathrm{eV}$, attesting to the presence of a dense and hot electron-hole plasma in the substrate

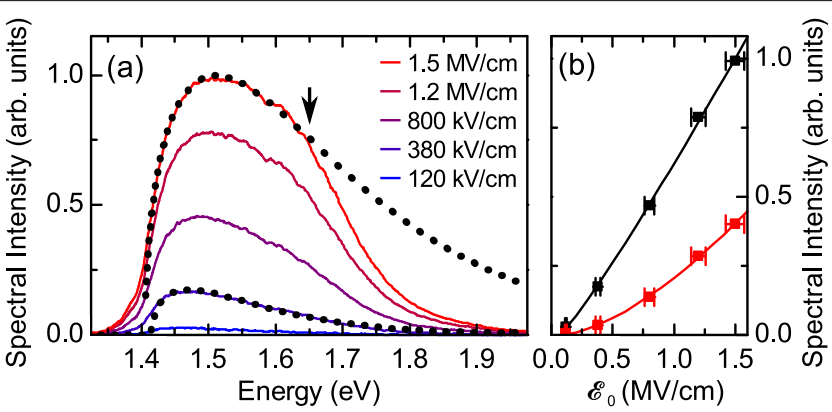

FIG. 3 (color online). (a) Luminescence spectra collected from sample B for different field amplitudes $\mathcal{E}_{0}$ (color-coded, solid curves). Black dotted curves: numerical fit functions (see text for details). (b) Scaling of EL intensity at photon energies $50 \mathrm{meV}$ (black) and $300 \mathrm{meV}$ (red) above the fundamental band gap with the terahertz field (squares). Solid curves: Power-law fit functions (see text).

[40]. At amplitudes above $800 \mathrm{kV} / \mathrm{cm}$, luminescence at yet higher photon energies of up to $\hbar \omega=1.9 \mathrm{eV}$ is observed. Additionally, a shoulder develops in the spectra at $1.65 \mathrm{eV}$ (black arrow). For an energy $50 \mathrm{meV}$ above the band gap, the luminescence intensity $I_{L}$ scales as $\left|\mathcal{E}_{0}\right|^{1.1}$. Still at high carrier excess energies of $300 \mathrm{meV}$ a low-order power law of $I_{L} \sim\left|\mathcal{E}_{0}\right|^{1.5}$ is found [Fig. 3(b)], characteristic of nonperturbative carrier generation [6].

For an estimate of the density of field-induced electron-hole pairs, we model the interband luminescence as a three-step process: (i) carrier generation within the duration of the $\mathrm{THz}$ transient, (ii) thermalization and cooling of the electron-hole plasma, and (iii) interband recombination. In high-purity, direct-gap semiconductors, the recombination time greatly exceeds the duration of carrier generation and thermalization. Therefore, we describe the electron-hole plasma by a hot carrier distribution and estimate an average carrier temperature $T$ and density $n$ by fitting the spectra using a convolution of a Fermi-Dirac distribution with the joint density of states in the effective mass approximation (Kubo-MartinSchwinger relation). This procedure works reasonably well up to $\mathcal{E}_{0}=380 \mathrm{kV} / \mathrm{cm}$, where we obtain $T \approx$ $1200 \mathrm{~K}$ and a sizable carrier concentration of $n=$ $5 \times 10^{18} \mathrm{~cm}^{-3}$. However, it cannot reproduce spectra featuring a prominent shoulder at $1.65 \mathrm{eV}$, as observed for higher field amplitudes. For large carrier excess energies, the effective mass approximation and the assumption of momentum-independent dipole moments falter and scattering into the $L$ valleys of GaAs sets in. Therefore, at $\mathcal{E}_{0}=1.5 \mathrm{MV} / \mathrm{cm}$, we restrict ourselves to fitting the EL spectrum in the energy range below $1.65 \mathrm{eV}$ [black dotted curve in Fig. 3(a)]. The best agreement with the data is obtained for an average electron temperature of $T \approx 2000 \mathrm{~K}$, a carrier concentration as high as $n=$ $1.5 \times 10^{19} \mathrm{~cm}^{-3}$, and an electron filling factor at the $\Gamma$ point of 0.8 , indicative of a degenerate carrier population. Hence, 
one may already attain population inversion in the most highly excited areas. Note that the above densities represent lower bounds of the actual peak values since the luminescence spectra are the result of spatial integration.

In order to understand the microscopic mechanism of terahertz-driven carrier generation in our structure, we refine our estimate of the near-field terahertz amplitude including near-field screening by the electron-hole plasma with $n=1.5 \times 10^{19} \mathrm{~cm}^{-3}$. To this end, we attribute a Drude conductivity to those subvolumes of the substrate that exhibit the highest near-field enhancement [25]. Although these simulations do show a reduction of the near-field amplitude, they still predict a maximum $\mathcal{E}_{n}>20 \mathrm{MV} / \mathrm{cm}$ [25]. The calculation underestimates the actual field since it assumes a time-independent carrier density that is already present at the leading edge of the terahertz pulse and neglects the dynamical buildup of screening [41]. We base our following considerations on a very conservatively chosen near-field amplitude of $\mathcal{E}_{n}=12 \mathrm{MV} / \mathrm{cm}$ for the intuitive picture sketched in Fig. 1(b), noting, however, that the actual fields are even larger.

In contrast to previous studies exploring field-driven EL in doped semiconductors $[13,23]$, our substrates are undoped. The initial pair generation, therefore, has to start from terahertz-induced interband transitions. Whether multiphoton ionization or interband tunneling prevails can be determined via the Keldysh parameter $\gamma_{K}=$ $\omega_{\mathrm{THz}} \sqrt{2 m E_{g}} / \mathrm{e} \mathcal{E}_{n}$ [42]. While the quantum nature of light dominates for $\gamma_{K} \gg 1$, tunneling injection becomes efficient for $\gamma_{K} \lesssim 1$. Strong terahertz-driven interband transitions in the regime of $\gamma_{K} \approx 0.13$ were recently demonstrated utilizing multi-MV/cm fields at $30 \mathrm{THz}$, and confirmed through a microscopic theory of interband polarization and intraband currents [6]. The electric peak field $\mathcal{E}_{n}$ in our present experiment approaches similar values as in Ref. [6], while $\hbar \omega_{\mathrm{THz}}=E_{g} / 390$ is substantially smaller. Therefore, we arrive at a record low $\gamma_{K} \approx 2 \times 10^{-2}$, fostering quasistatic Zener tunneling from the valence to the conduction band as an efficient first step of carrier generation. According to the rough estimate given above [Fig. 1(b)], Zener tunneling alone may already account for the carrier densities observed in the experiment [43]. Yet, we point out that our estimate is based on a band structure model [24] that is pushed to its limits in atomically strong fields.

In the absence of scattering, the terahertz bias would drive the wave vectors $k$ of Zener-injected carriers through the full Brillouin zone according to Bloch's acceleration theorem, ultimately giving rise to coherent Bloch oscillations [6]. We do not expect this scenario for the current situation since the wide conduction band of GaAs allows for efficient impact ionization once the carrier excess energy exceeds the threshold energy $E_{\mathrm{th}}=E_{g}\left(2 m_{e}+m_{\mathrm{hh}}\right) /$ $\left(m_{e}+m_{\mathrm{hh}}\right)=1.6 \mathrm{eV}$, where $m_{e}$ and $m_{\mathrm{hh}}$ are the electron and heavy-hole masses [13,24]. For $\mathcal{E}_{n} \approx 12 \mathrm{MV} / \mathrm{cm}$, electrons require less than $10 \mathrm{fs}$ to gain sufficient energy for a single impact ionization event. One half-cycle of the terahertz transient should thus drive a cascade of at least 10 ionization steps, multiplying the carrier density by $2^{10} \approx 10^{3}$ per oscillation half-cycle. The same multiplication factor can also be estimated considering that the ponderomotive energy $U_{p}=e^{2} \mathcal{E}_{n}^{2} / 4 m \omega_{0}^{2} \approx 2 \mathrm{keV} \approx 10^{3} E_{\mathrm{th}}$, and is hence sufficient to generate $10^{3}$ electron-hole pairs. Note that this multiplication effect can only fully apply as long as Pauli blocking does not limit this process. In fact, such saturation effects due to degenerate carrier populations should play an important role in our experiment and may account for the scaling of $I_{L}$ with a low power of $\mathcal{E}_{0}$ [Fig. 3(b)]. The regime explored here is in qualitative contrast to previous experiments where field ionization of shallow impurities by the far field of strong terahertz pulses has been used to observe luminescence with a field-independent narrow bandwidth $(\approx 5 \mathrm{meV})$, $I_{L} \sim \mathcal{E}_{0}^{8}$, and carrier densities between $10^{13}$ and $10^{16} \mathrm{~cm}^{-3}[13]$.

Besides the local field enhancement, metamaterials offer a second functionality: They couple the terahertz response of the microscopic volume hosting the dense electron-hole plasma back into the far field [22,34]. Figure 4 demonstrates
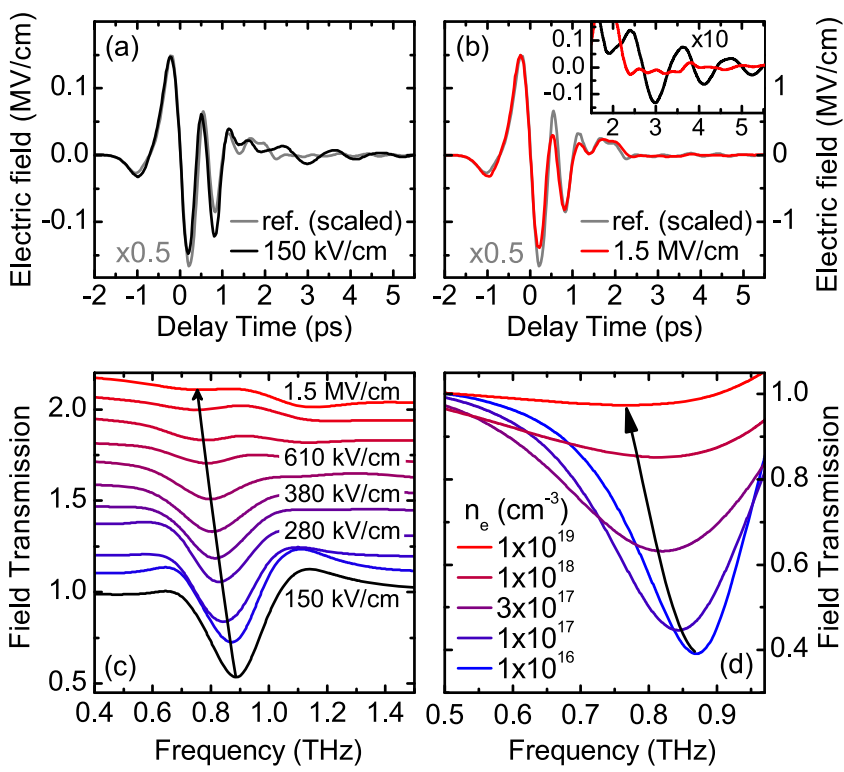

FIG. 4 (color online). Nonlinear THz transmission through the metamaterial sample. (a) Transmitted terahertz waveform (black curve) for a peak field of $\mathcal{E}_{0}=150 \mathrm{kV} / \mathrm{cm}$. (b) Corresponding transient for $\mathcal{E}_{0}=1.5 \mathrm{MV} / \mathrm{cm}$ (red curve). Gray curve: scaled waveform of incident $\mathrm{THz}$ transient. Inset: Magnified view of waveforms in (a) and (b). (c) Transmission spectra for different terahertz peak fields, vertically offset for clarity. For $\mathcal{E}_{0}=$ $150 \mathrm{kV} / \mathrm{cm}$ (black curve) a sharp transmission minimum at $0.88 \mathrm{THz}$ is observed, which redshifts (black arrow) and bleaches for increasing $\mathcal{E}_{0}$. (d) Numerical simulation of the spectral response of the structure for a series of carrier concentrations in the gap region using an electron mobility in GaAs of $\mu_{\mathrm{GaAs}}=2600 \mathrm{~cm}^{2} / \mathrm{Vs}$. Black arrows are guides to the eye 
that the extremely subwavelength interaction regions of the sample induce a complete suppression of the metamaterial resonance, causing a self-amplitude-modulation of the terahertz pulses. The transmitted waveform for $\mathcal{E}_{0}=$ $150 \mathrm{kV} / \mathrm{cm}$ [Fig. 4(a)] exhibits trailing oscillations characteristic of the resonant metamaterial response for delay times $t>2 \mathrm{ps}$. These features vanish completely if the terahertz peak amplitude is increased to $\mathcal{E}_{0}=1.5 \mathrm{MV} / \mathrm{cm}$ [Fig. 4(b)]. In the frequency domain this switch-off of the resonance manifests itself in a change of the relative field transmission from $50 \%\left(\mathcal{E}_{0}=150 \mathrm{kV} / \mathrm{cm}\right)$ to nearly unity $\left(\mathcal{E}_{0}=1.5 \mathrm{MV} / \mathrm{cm}\right)$, at a frequency of $0.88 \mathrm{THz}$ [Fig. 4(c)]. Simultaneously, the metamaterial resonance shifts from 0.88 to $0.75 \mathrm{THz}$.

The transients also illustrate the time scale on which the strong terahertz nonlinearity establishes itself. Transmitted waveforms follow the same time dependence as the incident transient up to a delay time of $t=0 \mathrm{fs}$ [Figs. 4(a) and 4(b)]. At the subsequent three field extrema, the transmitted amplitude is steadily reduced in the highfield limit [Fig. 4(b)] due to accumulative carrier generation occurring at a progressively increasing rate in each halfcycle of the terahertz pulse. We therefore expect that a significant reduction of the near-field amplitude by the dense electron-hole plasma occurs only for the later half-cycles where the majority of carriers are generated.

Using FDFD simulations we model the far-field terahertz response caused by carrier injection, assuming conductive slabs in the substrate below the capacitive gap region [25]. Calculated spectra are shown in Fig. 4(d) for carrier densities of $n=10^{16}-10^{19} \mathrm{~cm}^{-3}$. Since our simulation neither accounts for the temporal dynamics nor for the precise spatial density profile of the carrier population, the densities used here only mark a lower bound for the actual peak values. Despite these simplifications, the nonlinear response of the metamaterial is reproduced qualitatively well: With increasing carrier density, the $R L C$ circuit's loss rate rises and the oscillation is strongly damped, resulting in a reduction of the resonance frequency and a decrease of the oscillator strength.

In conclusion, we have demonstrated a new class of extremely nonperturbative nonlinearities in a semiconductor with an unprecedented ponderomotive potential reaching the $\mathrm{keV}$ range and a Keldysh parameter of only $\gamma_{K} \approx 2 \times 10^{-2}$. The strong fields allow us to inject degenerate electron-hole populations, leading to broadband interband electroluminescence at energies more than 500 times the terahertz photon energy, and nonlinear terahertz transmission through the sample. With increasing field amplitude the resonance of the metamaterial sample is redshifted and finally switched off completely. Exploiting nondestructive near-field enhanced terahertz biasing we reach record field amplitudes of at least $12 \mathrm{MV} / \mathrm{cm}$ for center frequencies below $1 \mathrm{THz}$. This result is an important step towards high-field studies for electronics at terahertz clock rates. For instance, the perspective to reach population inversion in custom-cut nanoscale regions makes our metamaterials an interesting platform for submicron optoelectronics at terahertz speeds. The strongly nonlinear terahertz response allows for novel devices such as terahertz transistors with femtosecond precision, spectrally selective switches, and low-threshold saturable absorbers, all flexible in frequency and bandwidth by the choice of metamaterial.

This work was supported by theEuropean Research Council through ERC Grants No. 305003 (QUANTUMsubCYCLE) and No. 280048 (ECOMAGICS) and the Deutsche Forschungsgemeinschaft through projects LA 3307 and collaborative research center SFB 689.

*christoph.lange@physik.uni-regensburg.de

[1] R. Ulbricht, E. Hendry, J. Shan, T. F. Heinz, and M. Bonn, Rev. Mod. Phys. 83, 543 (2011).

[2] T. Kampfrath, K. Tanaka, and K. A. Nelson, Nat. Photonics 7, 680 (2013).

[3] A. Sell, A. Leitenstorfer, and R. Huber, Opt. Lett. 33, 2767 (2008).

[4] F. Junginger, B. Mayer, C. Schmidt, O. Schubert, S. Mährlein, A. Leitenstorfer, R. Huber, and A. Pashkin, Phys. Rev. Lett. 109, 147403 (2012).

[5] M. Rini, R. Tobey, N. Dean, J. Itatani, Y. Tomioka, Y. Tokura, R. W. Schoenlein, and A. Cavalleri, Nature (London) 449, 72 (2007).

[6] O. Schubert, M. Hohenleutner, F. Langer, B. Urbanek, C. Lange, U. Huttner, D. Golde, T. Meier, M. Kira, S. W. Koch, and R. Huber, Nat. Photonics 8, 119 (2014).

[7] F. Blanchard, L. Razzari, H.-C. Bandulet, G. Sharma, R. Morandotti, J.-C. Kieffer, T. Ozaki, M. Reid, H. F. Tiedje, H. K. Haugen, and F. A. Hegmann, Opt. Express 15, 13212 (2007).

[8] W. Huang, E. Granados, W. R. Huang, K.-H. Hong, L. E. Zapata, and F. X. Kärtner, Opt. Lett. 38, 796 (2013).

[9] J. Hebling, G. Almasi, I. Kozma, and J. Kuhl, Opt. Express 10, 1161 (2002).

[10] H. Hirori, A. Doi, F. Blanchard, and K. Tanaka, Appl. Phys. Lett. 98, 091106 (2011).

[11] B. Zaks, R. B. Liu, and M. S. Sherwin, Nature (London) 483, 580 (2012).

[12] M. C. Hoffmann, J. Hebling, H. Y. Hwang, K.-L. Yeh, and K. A. Nelson, Phys. Rev. B 79, 161201 (2009).

[13] H. Hirori, K. Shinokita, M. Shirai, S. Tani, Y. Kadoya, and K. Tanaka, Nat. Commun. 2, 594 (2011).

[14] S. Tani, F. Blanchard, and K. Tanaka, Phys. Rev. Lett. 109, 166603 (2012).

[15] F. Blanchard, D. Golde, F. H. Su, L. Razzari, G. Sharma, R. Morandotti, T. Ozaki, M. Reid, M. Kira, S. W. Koch, and F. A. Hegmann, Phys. Rev. Lett. 107, 107401 (2011).

[16] N. Köster, A. Klettke, B. Ewers, R. Woscholski, S. Cecchi, D. Chrastina, G. Isella, M. Kira, S. Koch, and S. Chatterjee, New J. Phys. 15, 075004 (2013).

[17] W. D. Rice, J. Kono, S. Zybell, S. Winnerl, J. Bhattacharyya, H. Schneider, M. Helm, B. Ewers, A. Chernikov, M. Koch, 
S. Chatterjee, G. Khitrova, H. M. Gibbs, L. Schneebeli, B. Breddermann, M. Kira, and S. W. Koch, Phys. Rev. Lett. 110, 137404 (2013).

[18] J. Kono, M. Y. Su, T. Inoshita, T. Noda, M. S. Sherwin, S. J. Allen, and H. Sakaki, Phys. Rev. Lett. 79, 1758 (1997).

[19] T. Kampfrath, A. Sell, G. Klatt, A. Pashkin, S. Mährlein, T. Dekorsy, M. Wolf, M. Fiebig, A. Leitenstorfer, and R. Huber, Nat. Photonics 5, 31 (2011).

[20] T. Kubacka et al., Science 343, 1333 (2014).

[21] S. Fleischer, Y. Zhou, R. W. Field, and K. A. Nelson, Phys. Rev. Lett. 107, 163603 (2011).

[22] K. Fan, H. Y. Hwang, M. Liu, A. C. Strikwerda, A. Sternbach, J. Zhang, X. Zhao, X. Zhang, K. A. Nelson, and R. D. Averitt, Phys. Rev. Lett. 110, 217404 (2013).

[23] M. I. Nathan, G. Burns, S. E. Blum, and J. C. Marinace, Phys. Rev. 132, 1482 (1963).

[24] E. O. Kane, J. Phys. Chem. Solids 12, 181 (1959).

[25] See Supplemental Material at http://link.aps.org/ supplemental/10.1103/PhysRevLett.113.227401, which includes Refs. [26-33], for a discussion of Zener tunneling injection, near-field enhancement, and carrier dynamics in our structure.

[26] M. A. Seo, H. R. Park, S. M. Koo, D. J. Park, J. H. Kang, O. K. Suwal, S. S. Choi, P. C. M. Planken, G. S. Park, N. K. Park, Q. H. Park, and D. S. Kim, Nat. Photonics 3, 152 (2009).

[27] R. L. Olmon, B. Slovick, T. W. Johnson, D. Shelton, S.-H. Oh, G. D. Boreman, and M. B. Raschke, Phys. Rev. B 86, 235147 (2012).

[28] S. E. Ralph, Y. Chen, J. Woodall, and D. McInturff, Phys. Rev. B 54, 5568 (1996).

[29] J. Hebling, M. C. Hoffmann, H. Y. Hwang, K.-L. Yeh, and K. A. Nelson, Phys. Rev. B 81, 035201 (2010).

[30] F. Bloch, Z. Phys. 52, 555 (1929).

[31] P. Yu and M. Cardona, Fundamentals of Semiconductors: Physics and Materials Properties (Springer, Berlin, 2005).
[32] L. M. Smith, D. R. Wake, J. P. Wolfe, D. Levi, M. V. Klein, J. Klem, T. Henderson, and H. Morkoç, Phys. Rev. B 38, 5788 (1988).

[33] B. A. Ruzicka, L. K. Werake, H. Samassekou, and H. Zhao, Appl. Phys. Lett. 97, 262119 (2010).

[34] M. Liu, H. Y. Hwang, H. Tao, A. C. Strikwerda, K. Fan, G. Reiser, A. J. Sternbach, K. G. West, S. Kittiwatanakul, J. Lu, S. Wolf, F. G. Omenetto, X. Zhang, K. A. Nelson, and R. D. Averitt, Nature (London) 487, 345 (2012).

[35] Y.-G. Jeong, M. J. Paul, S.-H. Kim, K.-J. Yee, D.-S. Kim, and Y.-S. Lee, Appl. Phys. Lett. 103, 171109 (2013).

[36] J. R. Knab, A. J. L. Adam, M. Nagel, E. Shaner, M. A. Seo, D. S. Kim, and P. C. M. Planken, Opt. Express 17, 15072 (2009).

[37] M. Navarro-Cía, M. Natrella, F. Dominec, J. C. Delagnes, P. Kužel, P. Mounaix, C. Graham, C. C. Renaud, A. J. Seeds, and O. Mitrofanov, Appl. Phys. Lett. 103, 221103 (2013).

[38] T. L. Cocker, V. Jelic, M. Gupta, S. J. Molesky, J. A. J. Burgess, G. De Los Reyes, L. V. Titova, Y. Y. Tsui, M. R. Freeman, and F. A. Hegmann, Nat. Photonics 7, 620 (2013).

[39] W. Franz, Z. Naturforsch. 13a, 484 (1958); L. V. Keldysh, J. Exp. Theor. Phys. 33, 994 (1957) [Sov. Phys. JETP 6, 763 (1958)].

[40] Carrier drift and diffusion effects during and after the terahertz pulse, respectively, cause the EL map to slightly differ from the spatial carrier density profile (see Ref. [25]).

[41] R. Huber, F. Tauser, A. Brodschelm, M. Bichler, G. Abstreiter, and A. Leitenstorfer, Nature (London) 414, 286 (2001)

[42] L. V. Keldysh, J. Exp. Theor. Phys. 47, 1945 (1964) [Sov. Phys. JETP 20, 1307 (1965)].

[43] Note that luminescence of comparable intensity, spatial, and spectral shape is observed for all three samples, even when a thick $\mathrm{Al}_{2} \mathrm{O}_{3}$ tunnel barrier is inserted between metamaterial and substrate. This suggests that carrier injection through the Schottky barrier formed between the gold structure and the semiconductor substrate is not dominant. 